\title{
Telomerase activity in peripheral blood mononuclear cells after whole body irradiation
}

\author{
DIRK NEUHOF, MARC BISCHOF, JÜRGEN DEBUS and KLAUS-JOSEF WEBER
}

\author{
Department of Radiation Oncology, University of Heidelberg, Heidelberg, Germany
}

Received October 27, 2006; Accepted December 22, 2006

\begin{abstract}
Telomerase activity in vitro represents a radiationinducible function. To test this effect after irradiation in vivo, we measured telomerase activity in peripheral blood mononuclear cells (PBMC) of 25 patients with leukemia or lymphoma before and $1 \mathrm{~h}$ after whole body irradiation. Telomerase activity of the patients was compared to telomerase activity in PBMC of 15 healthy volunteers. Peripheral blood of the patients was taken before and $1 \mathrm{~h}$ after the first fraction of whole body irradiation ( $2 \mathrm{~Gy}$ ). Blood samples of the volunteers were irradiated ex vivo with 2 Gy. After FicollPaque density gradient centrifugation telomerase activity of $10^{4}$ PBMC per sample was measured using the telomerase PCR ELISA method. No age-dependence of telomerase activity was detected for both the volunteer and the patient group. Telomerase activity in patients was not statistically significantly increased compared to healthy individuals, and this parameter was also no prognostic factor for patient survival. After whole body irradiation an induction of telomerase activity was observed for only 7 patients (28\%), or in PBMC of 2 volunteers $(13 \%)$, respectively. In patients with radiation-inducible telomerase activity, a slightly better survival was indicated, but this difference did not reach statistical significance. The feasibility to assess in vivo radiation-induction of telomerase activity in PBMC of leukemia or lymphoma patients was demonstrated. An unexpectedly low number of whole body irradiated patients displayed this phenotype, and the treatment impact of telomerase upregulation in PBMC upon radiation exposure needs to be further analyzed.
\end{abstract}

\section{Introduction}

Telomerase is a reverse transcriptase that adds DNA repeats at chromosome ends. Human somatic cells usually lack telomerase activity and telomeres shorten with each division

Correspondence to: Dr Dirk Neuhof, Department of Radiation Oncology, University of Heidelberg, Im Neuenheimer Feld 400, 69120 Heidelberg, Germany

E-mail: dirk.neuhof@med.uni-heidelberg.de

Key words: telomerase, irradiation, peripheral blood mononuclear cells until the cells reach replicative senescence (1). By contrast, high levels of telomerase are expressed in the majority of human cancers (2). Stabilization of telomere length by telomerase, or less frequently by an alternative recombinational (ALT) pathway, appears to be a prerequisite for tumor cells to proliferate indefinitely (2). Some actively proliferative somatic cells, for example, hematopoietic stem cells (3), intestinal crypt cells (4) and basal cells in the epidermis (5), have weak but detectable telomerase activity. Telomerase activity is also detectable in human peripheral blood mononuclear cells (PBMC) and its activity is increased by antigen processing $(6,7)$. PBMC possess the capability for indefinite proliferation and the mechanism of clonal expansion to maintain sufficient immunoreactivity to antigens over lifespan. Telomerase activity in PBMC was reported to vary with age: the level of telomerase activity in PBMC of individuals aged $<20$ years was higher compared with that in individuals aged $\geq 20$ years (8). Furthermore, elevated telomerase activity in PBMC of leukemia patients compared to healthy controls has been shown, and was even related to disease progression (9-12).

Among other classes of DNA damage, ionizing radiation induces DNA double-strand breaks (DSB), which can lead to chromosome aberrations and cell death. Cells respond to DSB through a cascade of proteins ranging from sensors, which recognise the damage, through mediator proteins to a series of effectors that induce cell-cycle arrest, repair by homologous or nonhomologous mechanisms or cell death by apoptosis (13). In addition to telomere maintenance, telomerase can add telomeric sequences onto chromosome breaks at nontelomeric locations, a process termed chromosome healing $(14,15)$. Ionizing radiation-induced DSB provide a substrate for chromosome healing, but this appears to be a relatively rare event in mammalian cells (15). Nevertheless, a role of telomerase in the cellular response to radiation damage is supported by the observations from several laboratories that telomerase activity represents a radiation-inducible function (16-18).

Changes of telomerase activity after irradiation have been studied so far under in vitro conditions. To investigate telomerase activity after irradiation in vivo we measured telomerase activity in PBMC of 25 patients with leukemia or lymphoma undergoing whole body irradiation before bone marrow or blood stem cell transplantation. Telomerase activity of the patients was compared to telomerase activity in PBMC of 15 healthy volunteers. The PBMC of the volunteers were 
irradiated ex vivo. We evaluated changes of telomerase activity with age, differences between patients and healthy volunteers, induction of telomerase activity after irradiation, and telomerase activity as a possible prognostic factor for patients undergoing whole body irradiation.

\section{Materials and methods}

Heparinized peripheral blood $(3 \times 10 \mathrm{ml})$ was obtained by venopuncture from 15 healthy volunteers aged from 25 to 65 years old (median age: 34 years). The blood samples were irradiated ex vivo with 0 Gy (sham-treated) and 2 Gy using a medical linear accelerator operating in 6-MV X-ray mode at a dose-rate of $2.5 \mathrm{~Gy} / \mathrm{min}$. One hour after irradiation PBMC were isolated by Ficoll-Paque (Sigma Inc, St. Louis, USA) density gradient centrifugation. The PBMC were washed twice with phosphate-buffered saline (PBS) and stored at $-80^{\circ} \mathrm{C}$ until measurement of the telomerase activity.

To evaluate telomerase activity after irradiation in vivo 25 patients with leukemia or lymphoma undergoing whole body irradiation before bone marrow or blood stem cell transplantation were studied. All patients had received chemotherapy before the whole body irradiation, but never radiotherapy. The whole body irradiation is an established method in the preparation of patients to bone marrow or blood stem cell transplantation. The combination of an intensified chemotherapy and the whole body irradiation eliminates the malignant cell clone and suppresses the immune system to prevent a repulsion of the graft. The median age of the patients was 45 years, range 22 to 66 years. After agreement of the patients $10 \mathrm{ml}$ of heparinized peripheral blood was taken before the first irradiation. PBMC were purified on a Ficoll gradient, washed with PBS and stored at $-80^{\circ} \mathrm{C}$. Patients receiving whole body irradiation were irradiated twice daily with 2 Gy (medical linear accelerator, 6-MV X-ray mode) on three days to a whole dose of $12 \mathrm{~Gy}$. One hour after the first fraction ( $2 \mathrm{~Gy}) 10 \mathrm{ml}$ of heparinized peripheral blood was obtained. PBMC were purified on a Ficoll gradient, washed with PBS and stored at $-80^{\circ} \mathrm{C}$. Overall survival was calculated using the Kaplan-Meier method. Survival distributions were compared using the log-rank test (Statistica, StatSoft Inc, Tulsa, USA).

Telomerase activity was measured using the Telomerase PCR ELISA kit (Roche Diagnostics, Mannheim, Germany). The assay allows a nonradioactive detection of telomerase activity. In principle, telomerase activity, if present in cell lysates, adds telomeric repeats to a biotin-labeled primer. The elongation products are then amplified by PCR. An aliquot of the PCR product is denatured and hybridized to a digoxigenin-labeled, telomeric repeat-specific detection probe. This hybrid is immobilized by the biotin-labeled primer to a streptavidin-coated microtiter plate. Finally, the digoxigenin labels are detected with an anti-digoxigenin peroxidase. The probe is visualized by virtue of peroxidase-metabolizing tetramethyl benzidine to form a colored reaction product. Using a microtiter plate reader, color intensities are measured at $450 \mathrm{~nm}$ with a reference wavelength of $690 \mathrm{~nm}$. Samples of $10^{4}$ PBMC were analysed by the Telomerase PCR ELISA method, and derived as mean optical density from three separate determinations.

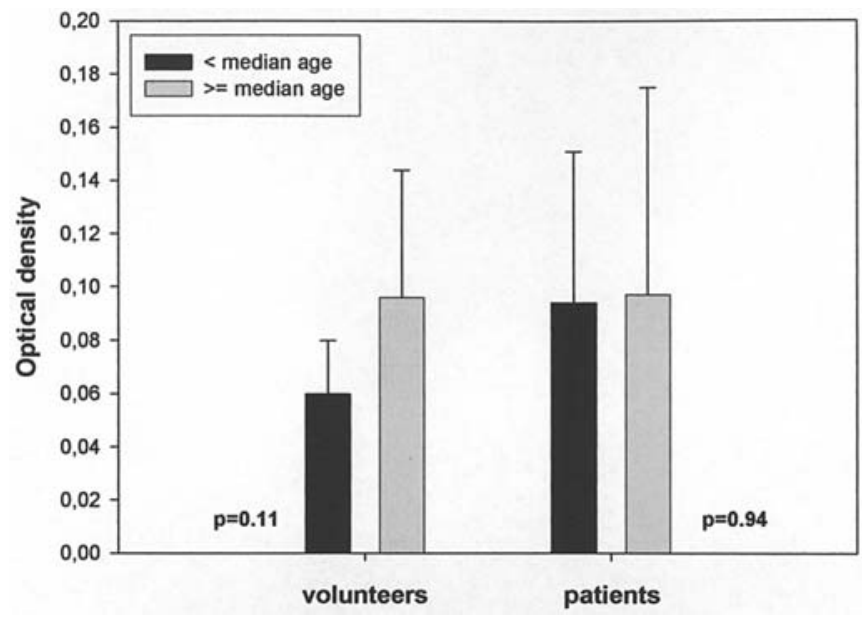

Figure 1. Mean telomerase activity of $10^{4}$ peripheral blood mononuclear cells of the volunteer and patient groups 'age < median age' and 'age $\geq$ median age'. Telomerase activity of the volunteers seemed to increase with age, but the difference was not significant $(\mathrm{p}=0.11)$. Telomerase activity of the patients was equal $(\mathrm{p}=0.94)$.

In previous in vitro experiments telomerase activity of unirradiated cells was used for an internal calibration curve, and the telomerase activity of the irradiated cells was converted to the cell equivalent of unirradiated cells (18). For the in vivo experiments of this study it is not possible to use an internal calibration curve. The levels of telomerase activity of the $\mathrm{PBMC}$ before and after in vivo irradiation have to be compared directly. To investigate the variability of the Telomerase PCR ELISA telomerase activity of $10^{4} \mathrm{PBMC}$ of four blood samples from one volunteer, taken in the morning and afternoon on two following days, was measured: the standard deviation was $9 \%$ of the mean value. Therefore an induction of telomerase activity after irradiation was defined as an elevation of at least $20 \%$ compared to the level before irradiation.

\section{Results}

To evaluate changes of telomerase activity with age the 15 healthy volunteers were divided into two groups: age $<34$ years (median age), and age $\geq 34$ years. Mean telomerase activity of $10^{4}$ PBMC of the two groups is given in Fig. 1. Mean optical density, as a measure of telomerase activity, of $10^{4}$ PBMC was 0.060 for the group 'age <34 years', and 0.096 for the group 'age $\geq 34$ years'. Telomerase activity seemed to increase with age, but the difference between the two groups was not significant $(\mathrm{p}=0.11)$. After ex vivo irradiation with $2 \mathrm{~Gy}$ an induction of telomerase activity was observed for two individuals $(13 \%)$.

Patient and treatment characteristics are shown in Table I. Of the 25 patients receiving whole body irradiation 11 patients had acute myeloid leukemia, 5 patients had acute lymphocytic leukemia, 4 patients had aggressive lymphoma, one patient had chronic myeloid leukemia, one patient had chronic lymphocytic leukemia, one patient had myelodysplastic syndrome, one patient had stage IV indolent lymphoma and one patient had stage IV Hodgkin lymphoma. The volunteer patients were divided by median age into two groups: age $<45$ years (median age), and age $\geq 45$ years. Mean optical density, as a measure 
Table I. Patient and treatment characteristics.

\begin{tabular}{|c|c|c|}
\hline Characteristic & No. & $\%$ \\
\hline \multicolumn{3}{|l|}{ Age (years) } \\
\hline Median & 45 & \\
\hline Range & $22-66$ & \\
\hline \multicolumn{3}{|l|}{ Sex } \\
\hline Male & 13 & 52 \\
\hline Female & 12 & 48 \\
\hline \multicolumn{3}{|l|}{ Primary diagnosis } \\
\hline AML & 11 & 44 \\
\hline ALL & 5 & 20 \\
\hline Aggressive lymphoma & 4 & 16 \\
\hline CML & 1 & 4 \\
\hline CLL & 1 & 4 \\
\hline MDS & 1 & 4 \\
\hline Indolent lymphoma, stage IV & 1 & 4 \\
\hline Hodgkin lymphoma, stage IV & 1 & 4 \\
\hline \multicolumn{3}{|l|}{ Remission status before RT } \\
\hline PR & 9 & 36 \\
\hline $\mathrm{CR}$ & 16 & 64 \\
\hline \multicolumn{3}{|l|}{ Transplantation } \\
\hline Autologous & 9 & 36 \\
\hline Allogeneic & 16 & 64 \\
\hline
\end{tabular}

AML, acute myeloid leukemia; ALL, acute lymphocytic leukemia; CML, chronic myeloid leukemia; CLL, chronic lymphocytic leukemia; MDS, myelodysplastic syndrome; RT, radiotherapy; PR, partial remission; $\mathrm{CR}$, complete remission.

of telomerase activity, of $10^{4}$ PBMC was 0.094 for the patient group 'age <45 years', and 0.097 for the patient group 'age $\geq 45$ years' ( $p=0.94$ ) (Fig. 1 ). The telomerase activity of the patients in comparison with the volunteers is shown in Fig. 2. No difference between these two groups was detected $(\mathrm{p}=0.43)$. Remission status before radiotherapy (partial response vs. complete response to chemotherapy) had no statistically significant influence on telomerase activity $(\mathrm{p}=0.61)$. After whole body irradiation with 2 Gy, 7 patients $(28 \%)$ showed an induction of telomerase activity in PBMC. This did not relate to preirradiation activity levels (three of these 7 patients had elevated telomerase activity before irradiation compared to the healthy volunteers). The patients with telomerase induction were not from one single leukemia group (5 patients had acute myeloid leukemia, one patient had chronic myeloid leukemia, and one patient had myelodysplastic syndrome).

The mean follow-up time of the patients was 9 months. A total of 7 patients $(28 \%)$ died. The 1-year survival rate was $65 \%$, the 2 -year survival rate $55 \%$ (Fig. 3). Remission status before radiotherapy had no impact on survival $(p=0.44)$. Patients who had high telomerase activity levels $(\geq 0.090$, median optical density) showed no statistically significant difference of survival rates compared to patients with low

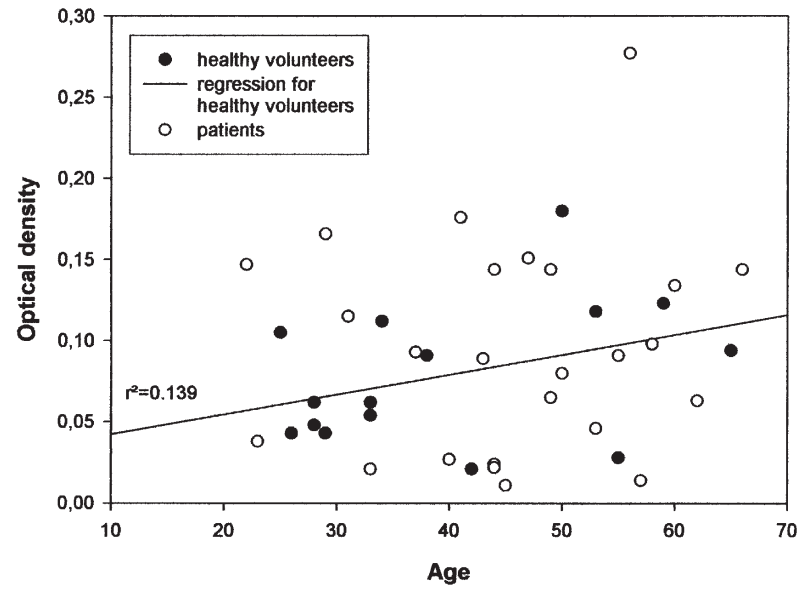

Figure 2. Telomerase activity of the 15 volunteers (black circles) and the 25 patients (white circles). To compare the telomerase activity of the patients with the telomerase activity of the volunteers the regression line of the telomerase activity levels of the volunteers was calculated $\left(r^{2}=0.139\right)$.

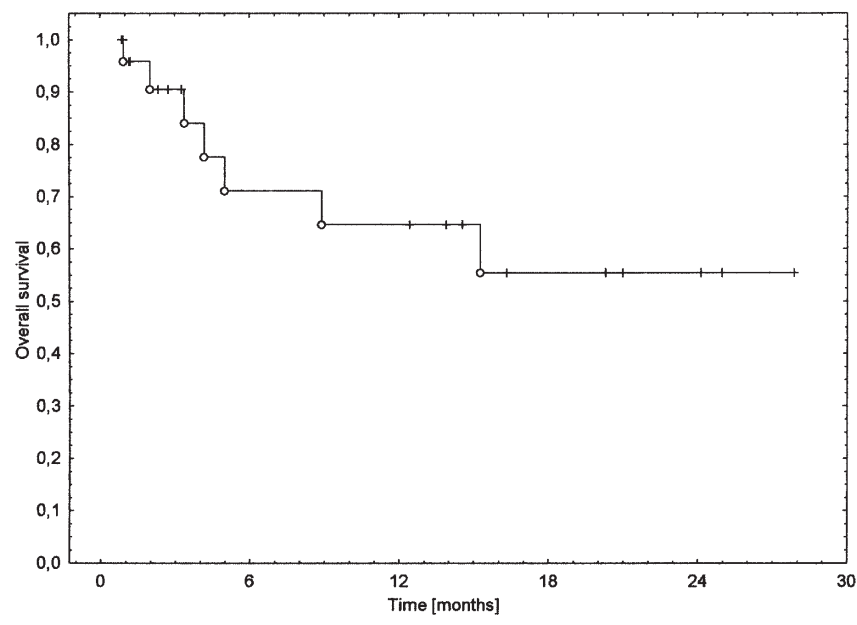

Figure 3. Overall survival of the 25 patients receiving whole body irradiation before bone marrow or blood stem cell transplantation. The 1-year survival rate was $65 \%$, the 2 -year survival rate was $55 \%$.

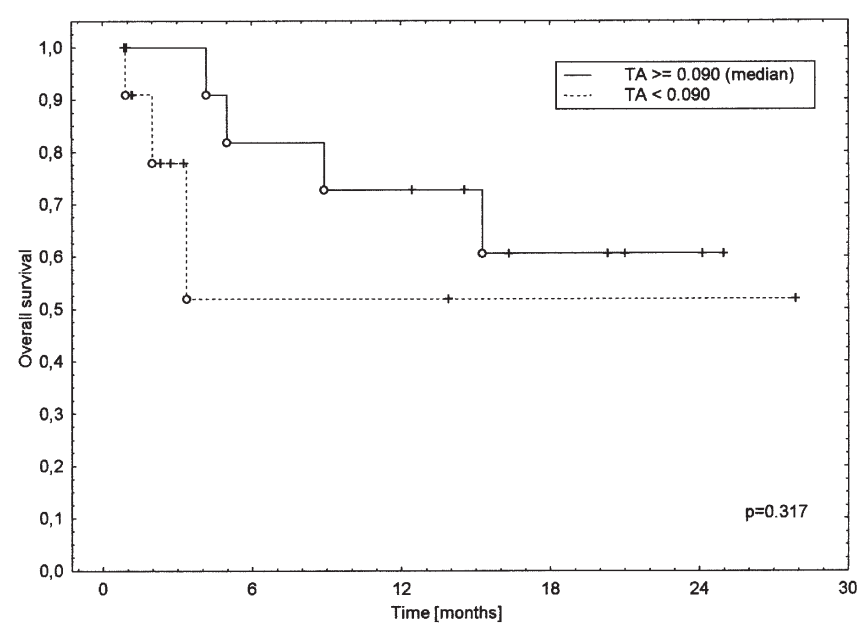

Figure 4. Overall survival of patients regarding level of telomerase activity before irradiation (telomerase activity $\geq 0.09$ vs. telomerase activity $<0.09$; median telomerase activity, 0.09). There was no statistically significant difference in survival $(\mathrm{p}=0.317)$. 


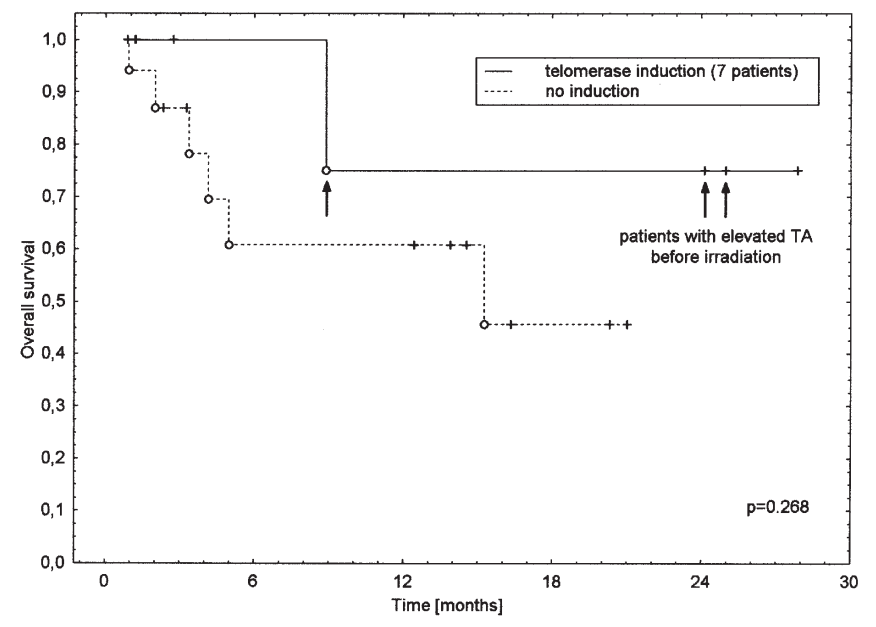

Figure 5. Overall survival of patients regarding induction of telomerase activity after 2 Gy whole body irradiation (telomerase induction vs. no telomerase induction). In patients with radiation-inducible telomerase activity, a slightly better survival was indicated, but this difference did not reach statistical significance $(\mathrm{p}=0.268)$. Three of the 7 patients with induction of telomerase activity had elevated telomerase activity before irradiation.

telomerase activity levels ( $\mathrm{p}=0.317$, Fig. 4). In patients with radiation-inducible telomerase activity, a slightly better survival was indicated, but this difference did not reach statistical significance ( $\mathrm{p}=0.268$, Fig. 5 ).

\section{Discussion}

In the present study, no age-dependence of telomerase activity in PBMC of healthy individuals (25 to 65 years old) was demonstrated, and the slight difference of mean telomerase activity between the groups 'age $<34$ years (median)' and 'age $\geq 34$ years' was not statistically significant. This result is contrary to those of previous studies: Iwama et al reported a decrease of telomerase activity in PBMC with age (8). However, their study contained younger individuals and higher telomerase activity in PBMC was observed for individuals aged $<20$ years compared to individuals aged $\geq 20$ years. Katayama et al also showed that telomerase activity declined with age in healthy individuals (aged 24-78 years), but not in patients with systemic lupus erythematosus (19). Telomerase activity in PBMC of patients with leukemia or lymphoma before undergoing whole body irradiation was also not dependent on patient age. Thus, it is presently not clear whether a general age-dependence of telomerase activity can be presumed.

A more important aspect of the present investigation was the possible elevation of telomerase activity in PBMC of patients with leukemia or lymphoma compared to healthy controls, which was reported for both adults (9-12) and children (20-22). An increase of telomerase activity was also inferred to relate to disease state, as a reflection of a progressively acquired capacity of tumor cells to sustain proliferation by this mechanism and/or of higher relative tumor cell burden $(9,10,12)$. It is still unclear, however, whether elevated telomerase activity is a prognostic factor for shortened survival with leukemias. For patients with adult T-cell leukemia high telomerase activity was associated with poorer prognosis
(23). Verstovsek et al showed that, for pediatric patients with acute myeloid leukemia, telomerase activity overexpression was a significant prognostic indicator for shortened survival (24). In contrast Ohyashiki et al found no correlation between telomerase activity and survival for adult patients with acute myeloid leukemia (10). For children with acute lymphoblastic leukemia telomerase activity was not associated with clinical outcome (25). In patients who achieved remission telomerase activity levels decreased to normal but were significantly higher at the time of recurrence or disease progression compared with telomerase activity levels at the time of diagnosis $(9,10,12)$. In our study, telomerase activity in leukemia or lymphoma patients did not exhibit a statistically significant increase compared to healthy controls, and this parameter was also no prognostic factor for the survival of patients. Considering the substantial interindividual variation of telomerase activity in the patients as well as in the healthy volunteers with large overlap between these groups, a delineation of a difference between them, if in fact present at all, would certainly require a much higher number of individuals being tested. Similarly, the significance of elevated telomerase activity as putative prognostic factor may only become detectable with much larger cohorts of patients, but with respect to the present result of a close similarity of such activity levels in leukemia patients with those of the healthy controls, the prognostic value of this parameter, even if explored to reach statistical significance in a large cohort, remains questionable.

One major focus of the present investigation was, for the first time, to assess a possible induction of telomerase activity in PBMC upon (whole body) irradiation in vivo. Several in vitro studies revealed that telomerase activity represents a radiationinducible function (16-18), even at low radiation doses and persisting over many cell generations (18). Based on the abovementioned reports of a possible relation between telomerase activity levels and leukemia patient survival, we reasoned, as a working hypothesis, that i) telomerase activity might also be induced in PBMC due to 2 Gy whole body irradiation, and ii) that this induction could impact on disease progression when cancers recur from surviving cancer cells with telomerase activity being elevated. Contrary to our expectation, radiation induction of telomerase was detectable in only $28 \%$ (7 out of 25 ) of the patients and $13 \%$ of the healthy individuals (criterion: increase $>2$ standard deviations of the interexperimental variability; see Materials and methods), and the magnitude of telomerase upregulation was in all these cases much lower than the respective results with diverse in vitro systems [e.g. $\leq 4$-fold in human lymphoblastoid cells (18)]. One could speculate that induction of telomerase activity occurred in patients that had low baseline activity (i.e. prior to irradiation), but 3 out of the 7 patients with radiation-induced activity already had preirradiation levels above the mean. This failure to detect a more frequent (among individuals) upregulation of telomerase in PBMC obviously prevented a survival analysis in relation to the extent of telomerase activation (in this subgroup). Also the survival of the radiation-inducible group was not statistically significantly decreased compared to the non-responders, whereby this limited data set rather showed a slightly better survival of the patients with radiationinducible telomerase activity. Further study will be necessary to clarify this important question. In this respect, the still 
unknown biological role of radiation induction of telomerase activity needs to be addressed. The ability of telomerase to add telomeric sequences at nontelomeric locations has conceivably been demonstrated $(14,15)$. This process could represent an important cofactor in the cellular DNA damage response by converting radiation-induced DNA double-strand breaks into stable chromosome breaks (chromosome healing), thus preventing DSB rejoining (14), or more importantly, misrejoining that could give rise to chromosomal rearrangements. While such considerations are still speculative, this type of damage response could potentially enhance the efficacy of radiation treatment aimed at eradicating tumor cells.

This study investigated for the first time telomerase activity in human PBMC after irradiation in vivo. The feasibility to assess in vivo radiation-induction of telomerase activity in PBMC of leukemia or lymphoma patients was demonstrated. An unexpectedly low number of whole body irradiated patients displayed this phenotype, and the treatment impact of telomerase upregulation in PBMC upon radiation exposure needs to be further analyzed.

\section{References}

1. Blackburn EH: Structure and function of telomeres. Nature 350: $569-573,1991$.

2. Kim NW, Piatyszek MA, Prowse KR, Harley CB, West MD, Ho PLC, Coviello GM, Wright WE, Weinrich SL and Shay JW: Specific association of human telomerase activity with immortal cells and cancer. Science 266: 2011-2015, 1994.

3. Chiu CP, Dragowska W, Kim NW, Vaziri H, Yui J, Thomas TE, Harley CB and Lansdorp PM: Differential expression of telomerase activity in hematopoietic progenitors from adult bone marrow. Stem Cell 14: 239-248, 1996.

4. Hiyama E, Hiyama K, Tatsumoto N, Kodama T, Shay JW and Yokoyama T: Telomerase activity in human intestine. Int $J$ Oncol 9: 453-458, 1996.

5. Haerle-Bachor C and Boukamp P: Telomerase avtivity in the regenerative basal layer of the epidermis in human skin and in immortal and carcinoma-derived skin keratinocytes. Proc Natl Acad Sci USA 93: 6476-6481, 1996.

6. Son NH, Murray S, Yanovski J, Hodes RJ and Weng NP: Lineage-specific telomere shortening and unaltered capacity for telomerase expression in human $\mathrm{T}$ and $\mathrm{B}$ lymphocytes with age. J Immunol 165: 1191-1196, 2000.

7. Weng NP, Levine BL, June CH and Hodes RJ: Regulated expression of telomerase activity in human $\mathrm{T}$ lymphocyte development and activation. J Exp Med 183: 2471-2479, 1996.

8. Iwama H, Ohyashiki K, Ohyashiki JH, Hayashi S, Yakata N, Ando K, Toyama K, Hoshika A, Takasaki M, Mori M and Shay JW: Telomeric length and telomerase activity vary with age in peripheral blood cells obtained from normal individuals. Hum Genet 102: 397-402, 1998.
9. Engelhardt M, Mackenzie K, Drullinsky P, Silver RT and Moore MA: Telomerase activity and telomer length in acute and chronic leukemia, pre- and post-ex vivo culture. Cancer Res 60: 610-617, 2000.

10. Ohyashiki JH, Ohyashiki K, Iwama H, Hayashi S, Toyama K and Shay JW: Clinical implications of telomerase activity levels in acute leukemia. Clin Cancer Res 3: 619-625, 1997.

11. Shay JW, Werbin H and Wright WE: Telomeres and telomerase in human leukemias. Leukemia 10: 1255-1261, 1996.

12. Xu D, Gruber A, Peterson C and Pisa P: Telomerase activity and the expression of telomerase components in acute myelogenous leukemia. Br J Haematol 102: 1367-1375, 1998.

13. Jackson SP: Sensing and repairing DNA double-strand breaks. Carcinogenesis 23: 687-696, 2002.

14. Murnane JP and Yu L: Acquisition of telomere repeat sequences by transfected DNA integrated at the site of a chromosome break. Mol Cell Biol 13: 977-983, 1993.

15. Slijepcevic P and Bryant PE: Chromosome healing, telomere capture and mechanisms of radiation-induced chromosome breakage. Int J Radiat Biol 73: 1-15, 1998.

16. Hande MP, Lansdorp PM and Natarajan AT: Induction of telomerase activity by in vivo X-irradiation of mouse splenocytes and its possible role in chromosome healing. Mutat Res 404: 205-214, 1998.

17. Hyeon Joo O, Hande MP, Lansdorp PM and Natarajan AT: Induction of telomerase activity and chromosome aberrations in human tumour cell lines following X-irradiation. Mutat Res 401: 121-131, 1998

18. Neuhof D, Ruess A, Wenz F and Weber KJ: Induction of telomerase activity by irradiation in human lymphoblasts. Radiat Res 155: 693-697, 2001.

19. Katayama Y and Kohriyama K: Telomerase activity in peripheral blood mononuclear cells of systemic connective tissue diseases. J Rheumatol 28: 288-291, 2001.

20. Engelhardt M, Ozkaynak MF, Drullinsky P, Sandoval C, Tugal O, Jayabose $\mathrm{S}$ and Moore MA: Telomerase activity and telomere length in pediatric patients with malignances undergoing chemotherapy. Leukemia 12: 13-24, 1998.

21. Januszkiewicz D, Wysoki J, Lewandowski K, Pernak M, Nowicka K, Rembowska J and Nowak J: Lack of correlation between telomere length and telomerase activity and expression in leukemic cells. Int J Mol Med 12: 935-938, 2003.

22. Malaska J, Sklenickova M, Krejci K, Fajkusova L, Bajer M, Hrstkova $\mathrm{H}$ and Fajkus J: Telomerase activity and epression and telomere analysis in situ in the course of treatment of childhood leukemias. Blood Cells Mol Dis 26: 534-539, 2000.

23. Kubuki Y, Suzuki M, Sasaki H, Toyama T, Yamashita K, Maeda K, Matsuoka H, Okayama A, Nakanishi T and Tsubouchi M: Telomerase activity and telomere length as prognostic factors of adult T-cell leukemia. Leuk Lymph 46: 393-399, 2005.

24. Verstovsek S, Manshouri T, Smith FO, Giles FJ, Cortes J, Estey E, Kantarjian H, Keating M, Jeha S and Albitar M: Telomerase activity is prognostic in pediatric patients with acute myeloid leukemia: comparison with adult acute myeloid leukemia. Cancer 97: 2212-2217, 2003.

25. Kleideiter E, Bangerter U, Schwab M, Boukamp P, Koscielniak E, Klotz U and Greil J: Telomeres and telomerase in paediatric patients with T-cell acute lymphoblastic leukemia (T-ALL). Leukemia 19: 296-298, 2005. 